\section{"Predictive Medicine" - the unpredictable story of a project}

\author{
by Koos van der Bruggen, Rathenau Insti- \\ tute
}

The Rathenau Institute has carried out a project on "Predictive Medicine" focusing primarily on the political and ethical "dilemmas" associated with predictive medicine. The main hypothesis of the study is that because of predictive medicine the generally accepted normative model based on patient autonomy should no longer be maintained. The hypothesis was strongly opposed and the project led to severe criticism of the Rathenau Institute. The reasons behind this apparent "failure" are analysed and interpreted.

\section{Introduction}

The Rathenau Institute set up the project on "Predictive Medicine" to gain more insight into the normative aspects of predictive medicine, and the way in which information on possible future ailments can effect the self-image of people.

In its project the Rathenau Institute had the goal to describe important technological developments in the field of Predictive Medicine, and also possible political and social consequences. Furthermore it was the intention to organise some debates with experts but also lay people to discuss the results and the possible social and political implications.

Questions that were to be treated in the project were:

- What predictive health knowledge does exist? Is this knowledge such that one can speak of predictive medicine?

- What are the possible social, political and economic consequences of such knowledge? How must these consequences be appreciated? Are consequences anticipated?

- Which organisations and institutions are involved in the development of predictive medicine? Which goals and visions do they have? What are their mutual relationships and interests?
- How is government involved?

\section{Predictive medicine}

Making predictions is inherent to medicine. But predictive medicine is a rather new phenomenon, because now not only the process of a disease can be predicted, but also the occurrence of a disease. The range of predictive information will grow rapidly because of developments such as:

- The results of the Human Genome Project: recently the complete human genome map was published (Science 2001);

- The combination of biotechnology with information technology, e.g. resulting in so called DNA-chips;

- The development of pharmacogenetics and the resulting growth of preventive medic ation (e.g. for osteoporosis).

It is still difficult to formulate a good definition of predictive medicine. Predictive medicine has the following characte ristics:

- It is not oriented towards the diagnosis of actual illness or treatment, but to the detection and reduction of risks;

- Predictive medicine therefore has a different time-scheme compared to symptomrelated medicine. It is often related to symptoms and illnesses that could manifest themselves far into the future;

- It assumes a statistical style of reasoning and the concomitant practices in the application of knowledge.

In brief, predictive medicine is about nonsymptom-related, risk-oriented health care practices. Its emergence indicates not merely a cognitive development in medicine but introduces new types of medical practice and the accompanying new relationships between doctor and patient, and between the medical profession and other institutions such as government and private insurance companies. Predictive medicine implies a significant shift in the societal roles of medicine and in the significance of medicine in the practice of citizenship. The project of the Rathenau Institute focused primarily on the political and ethical dilemmas that are intrinsically associated with predictive medicine. 


\section{The project}

As a start of the project an exploratory study was set up. Three researchers (2 sociologists and a philosopher) stated as a hypothesis that because of predictive medicine the generally accepted normative model based on patient autonomy perhaps cannot and should not be maintained in all aspects. Handling with risk asks for other normative models (de Vries, Horstman, Haveman 1997).

This preliminary conclusion was presented in a workshop with medical, genetic and ethical specialists. It appeared that most of them did not agree with the hypothesis and just emphasised the advantages of the autonomy model, also in new developments.

The Rathenau Institute asked the authors (De Vries, Horstman and Haveman) to elaborate this hypothesis further in a more extensive study.

Furthermore, research was done on the technological state of the art in predictive medicine: what are realistic expectations in the field of predictive medicine?

As a third part of the project scenarios were written, in which possible situations egarding predictive medicine in the year 2010 were described.

Four scenarios were developed based on the dimensions technical progress, rapid or otherwise (i.e. the ability to gain insight into the possibility and the risks of contracting a particular illness) and the extent to which this knowledge is used by the individual. The various scenarios address the consequences for the role of government and financial institutions, and for the organisation of the health care sector. The scenarios show a marked difference between two possible situations. The first situation is that in which individuals almost routinely have their genetic profile determined and, by taking food supplements and undergoing genetic therapy, attempt to prevent genetic ailments from manifesting themselves. The second situation is that in which the overriding opinion is that an individual's knowing that there is a risk of illness does more harm than good to the quality of life - especially when there is no adequate treatment. The two scenarios make it clear that, in the implementation of predictive medicine, there will be a question of striking a balance between the possibility of using the knowledge of the risk of illness and the associated stress and the false security given by the diagnostic tests.

The intention was that the scenarios could be used as an instrument for a number of $d-$ bates, with scientists and other involved persons as well as with lay people (Kruijff, Schreuder 1999).

But things went completely different! It appeared that the reaction of the "medical world" to the elaborate study of De Vries, Horstman and Haveman was even more critical and rejecting than their comment on the preparatory study. This became clear in the Advisory Group the Rathenau Institute had formed. This group consisted of medical, ethical and other professionals. It became clear that this group was divided. Especially the medical and genetic specialists were very angry about the (preliminary) results of the normative-political study of De Vries, Horstman and Haveman. They saw it as an insult for their profession; they accused the authors and later also the Rathenau Institute of ignorance and even malicious intent.

What were the most important results of the study? The core reasoning in the study was (again) that predictive medicine is different in character from symptom-related medicine. When there are symptoms, there is a clearly defined physical or psychological deficiency, and there is a clear relationship between the treatment and the symptom. In predictive medicine, the situation is considerably more complex: someone who feels a hundred percent fit is confronted with the knowledge of risks to his or her health. This can cause major changes in life-style, in expectations of the future and in social relationships. The principle of autonomy, which allows the patient to decide whether or not to undergo treatment, does not work very well in predictive medicine. This is because the implications of predicting an ailment that will only manifest itself in the future can, after all, not be foreseen, but they can be far reaching. What is more, the results of predictive medicine can have - unforeseen - societal and social-cultural consequences. Not only does the person involved have to cope with the know ledge of a future illness, society will also react to this knowledge. How do institutions treat 
someone of whom it is known that he or she runs a risk of contracting a serious illness? What "status" does such a person have within the society? And which demands do his or her social surroundings impose on the life-style of the person involved? The consequences of predictive medicine can go considerably further than the four walls of the doctor's consulting room. Because of that the authors argue that not only "classical" medical-ethical criteria should be taken into account when judging new developments, but also moral considerations on "how to live". The principle of autonomy of the patient does not provide enough protection, thus - according to the study - active political measures are necessary. They make a plea for new concepts of citizenship and of a decent society. By developing these concepts government can contribute to the introduction and further development of predictive medicine without negative consequences for certain groups or individuals in society. An agenda for political action that can create guarantees and protection is necessary in the light of the further development of predictive medicine (see Box 1).

Box 1: Agenda for political action (Horstman, de Vries, Haveman 1999)

I. Measures that promote the openness of public discourse in respect to the political aspects of predictive medicine.

1. Public attention to and explicit investigation into divergent aspects of a risk culture: gender, class and ethnicity, changes in perspectives of the future, family relationships, relationships between generations etc.

2. Development of a political conceptual framework for health care and medicine as being in service of the citizenry and research into citizenship in a risk culture.

3. Promotion of the public airing of points of dissension between medical experts in the field of predictive medicine. Demolishing the idea that medicine is a monolithic structure. Protection of warning voices in medicine and health care.

4. Promoting plurality in respect to predictive medicine: making explicit in the mass media and education (including medical education) variations in experiences - personal accounts, points of view.

5. Regulation of the formal position of citizens in expertise monopolies, for instance, in scientific committees, ethical committees. Development of new accountability forums. Public supervision of professional self-supervision.

6. Monitoring of quality control in health care (including health education and information) from the point of view of citizenship, plurality and autonomy.

7. Introduction of the rule that in scientific articles there should be accountability not only for the methodology employed but also for the ethical decisions entailed in respect to the responsibility involved - ensuring autonomy, informed consent etc.

II. Measures to introduce checks and balances and "fire escapes".

These measures are chiefly in respect to the prevention or reduction of detrimental (accumulative) effects in respect to sections of the population as a result of the developments in predictive medicine.

1. Constitutional guarantees that people can be reasonably insured.

2. Control of data banks and trajectories that contain and supply medical data.

3. Control of the borderline between medical investigation and clinical practice.

4. Control of the borderline between health care research into risks and social policy.

5. Control of the borderline between various fields of insurance.

6. Control of the borderline between the insurance and the employment market. 
The medical and genetic specialists in the Advisory Group did not see the risks of these perhaps unintended political and social consequences of the development of predictive medicine. It appeared impossible to come to a kind of compromise about the content.

The Rathenau Institute concluded that in such a situation a broader societal debate was not useful. Instead of that a closed workshop was organised to which both "parties" and other more or less involved persons were invited. The goal of this meeting was to get clear what was the essential point of discussion, because we as Rathenau Institute had the impression that both parties just did not enough understand each other. The workshop was a complete failure, in which the antagonism was only sharpened.

After the debate the Rathenau Institute had to think about the consequences. On great lines we agreed with the authors of the study and we were willing to publish it. But this would be harmful, if the whole medical world would oppose to it. The Rathenau Institute would loose its goodwill.

We asked some neutral experts for second and third opinions. Most of the reactions were very positive. And after a long time of discussion the staff and the Board of the Rathenau Institute decided to publish the results (Horstman, de Vries, Haveman 1999). Moreover the Rathenau Institute published its own "Message to Parliament" (Rathenau Institute 2000), in which we explained why the questions that were raised regarding the possible social consequences of predictive medicine were politically relevant. Except for some more practical points the main argument in the Message to Parliament was on the meaning of citizenship in an era of predictive medicine (see Box 2).

Box 2: Conclusion from Message to Parliament (Rathenau Institute 2000)

Predictive medicine is one of the developments that can contribute to a blurring of the borderlines between health care and other domains. Consequence will be that the roles of "citizen" and "patient" are no longer strictly separated. The citizen will not only be patient in the consulting room of the doctor, but he or she can be considered as a client of the care-system at any moment and in relationship to all his or her actions.

The present system of checks and balances in health care is based on the relation between patient and care provider. This situation is the basis of regulation and ethical considerations. Because of the blurring borderlines between health care and other domains because of predictive medicine this basis will be no longer valid. The existing regulations and current considerations in medical ethics do not give an answer to existential questions as how to cope with knowledge of your future health, or how to be responsible for your future when choosing a partner or having or not having children.

The consequence of the blurring borderline between a specific medical context and "life itself" is that it is less adequate to use the terminology of patient. In fact the "citizens" are the ones that have to be addressed. The citizens have to give answers to new questions, the citizens must fit the new possibilities and their consequences into their lives. This does not only affect the citizen as an autonomous individual but also as part of a social structure and a social history. From this perspective also the roles of the government and the involved parties in health care are changing.

These developments lead to new demands for good citizenship. It can be seen as a task for government to monitor the development of all necessary conditions for good citizenship. So government must guarantee good training on the field of predictive medicine. Risk education should be a part of this new training.

The results were presented in a meeting with members of parliament from different political parties. Most of them regarded the results of the project as a useful contribution to the dbate on predictive medicine and more special genetics. But one of the genetic experts (a member of the advisory commission) spoke of "a black day" for the Rathenau Institute. We were happy that a prominent member of parliament immediately denied this accusation. 
The presentation draw some attention of radio and television, this also because in our Message to Parliament we had given some concrete examples of possible consequences of predictive medicine.

\section{Some conclusions}

Looking back at the project Predictive Medicine from the perspective of the Rathenau $\mathbf{h}$ stitute as a parliamentary TA institute, the following conclusions can be drawn:

- A project that takes a different course than is planned, is not because of that a failure; on the contrary it proved that predictive medicine was a subject which raised a lot of new and other questions than the Rathenau Institute expected;

- We had a big advisory group in which all possible antagonisms were built in: that proved to be not a workable construction;

- Conflicts should not be sought, but it is also not useful to avoid them at all costs; perhaps the discussion around predictive medicine is the forerunner of a coming paradigm discussion in and around medicine;

- There is a great difference in culture between many medical and genetical specialists and philosophers and sociologists, but also politicians.

\section{References}

de Vries, G. H.; Horstman, K.; Haveman, O., 1997 : Politiek van preventie. Normatieve aspecten van voorspellende geneeskunde. The Hague: Rathenau Instituut. Working Document 58

Horstman, K.; de Vries, G. H.; Haveman, O., 1999: Gezondheidspolitiek in een risicocultuur. Burgerschap in het tijdperk van de voorspellende geneeskunde. The Hague: Rathenau Institute. Study 38, p. 141-142

Kruijff, A. F.; Schreuder, R. F., 1999: Toekomstscenario's voorspellende geneeskunde. The Hague: Rathenau Institute. Working Document 73 Rathenau Institute, 2000: Goede tijding, slechte tijding (Good message, bad message). Bericht aan het Parlement. The Hague

Science 2001 Feb. 16;291(5507):1304-1351.

\section{Contact}

Koos van der Bruggen

Rathenau Institute

P.O. Box 85525, NL-2508 CE The Hague

Tel.: + 31 (0) 703421542

Fax: + 31 (0) 703633488

e-mail: K.vanderBruggen@Rathenau.knaw.nl

Homepage: http://www.rathenau.knaw.nl

\section{POST study on Pharmaco- genetics}

\author{
by David Cope and Peter Border, Parliamen- \\ tary Office of Science and Technology
}

Genetics is an important factor in the overall impact of a person's reaction to a specific drug. With increasing knowledge about the human genome, pharmacogenetic testing will become more important, as it opens up the prospect of personalised medicine, where each patient receives the treatment most appropriate to him. It poses however also challenges to regulators and raises questions about the nature of the information derived by phamacogenetic profiling.

\section{What is pharmacogenetics?}

It has long been known that different people may react differently to the same drug. Indeed, a rule of thumb has evolved in the pharmaceutical industry, that only around one in three people given a particular drug will benefit from it. The remaining two thirds either will not respond to the drug at all, or may suffer an adverse reaction.

Pharmacogenetics is an attempt to explain this variation in the way individuals respond to drugs in terms of genetics. This is not to suggest that genetics is the only factor involved. An individual's response to a drug will also be influenced by a range of other (environmental) factors such as what and when they last ate. Other factors such as compliance with a drug regime (the extent to which an individual takes the right drug at the right time) will also contribute to the overall impact of the drug. But it 